\title{
Anesthesia Screening for Obstructive Sleep Apnea
}

\author{
Tim Brady ${ }^{1}$, Jordan Rohrich ${ }^{2}$ \\ Staff Nurse Anesthetist \\ Catholic Health Initiative Creighton University Medical Center Omaha, Nebraska \\ ${ }^{1}$ Tj77brady@yahoo.com, ${ }^{2}$ Jordan.rohrich@gmail.com
}

\begin{abstract}
In the United States, an estimated $82 \%$ of men and $92 \%$ of women with moderate to severe obstructive sleep apnea (OSA) have not been diagnosed. ${ }^{l}$ Combined with the fact that Americans' waist lines are expanding, it is expected that these numbers will increase. This study takes a well-known obstructive sleep apnea screening questionnaire, STOP-BANG, and attempts to identify a positive test score with potential risks and complications for surgical patients requiring anesthesia. In addition, this study attempts to look at the relationship between the patients' BMI and intraoperative and postoperative complications. This study also attempts to determine if STOP-BANG-positive patients require longer postoperative recovery times. Anesthesia providers care for many patients who may fit the profile of having OSA. Undiagnosed OSA patients may potentially place themselves at a higher risk for complications during the perioperative period. It is beneficial for those patients to be flagged with utilization of a preoperative assessment tool such as STOP-BANG. By identifying the at-risk patients early, measures can be taken to increase safety and improve outcomes.
\end{abstract}

Keywords: Obstructive Sleep Apnea, STOP-BANG, Anesthesia, Screening, OSA.

\section{INTRODUCTION}

The Greek word "apnea" means "without breath." Obstructive sleep apnea (OSA) was first described in scientific literature in 1966. An estimated $82 \%$ of men and $92 \%$ of women with moderate to severe OSA have not been diagnosed. ${ }^{1}$ Combined with the fact that Americans' waist lines are expanding, it is expected these numbers will increase. OSA is the repetitive obstruction of the upper airway resulting in oxygen desaturation and arousal from sleep. These events are defined as complete cessation or marked reduction in air flow. ${ }^{2}$ There are two types of sleep apnea: central and obstructive. In central sleep apnea, there is an acute onset of irregular breathing that is unresponsive to chemical or vagal stimuli (no voluntary effort), which is common when patients are under anesthesia. Respiratory drive also is often depressed during sleep. With obstructive sleep apnea, respiratory effort occurs with no subsequent air flow and is commonly perceived as snoring. ${ }^{3}$

Patients with OSA have higher incidences of hypoxia, dysrhythmias, hypertension, coronary artery disease, congestive heart failure, myocardial ischemia, pulmonary hypertension, cerebrovascular accidents, and esophageal reflux. In addition, anesthetic agents and opioids have adverse effects on the ventilator control centers of the brain and upper airway muscle tone. ${ }^{5,6}$ If patients go under anesthesia undiagnosed, their chances of experiencing postoperative complications may increase. It is imperative to identify patients undergoing anesthesia who are at risk for OSA. ${ }^{4,7}$

There are multiple research papers on the subject of OSA, including screening tools, identifying OSA patients, and intraoperative and postoperative outcomes of those either clinically diagnosed with OSA or those presumed through a questionnaire. According to the Vasu, et al (2010), "The high rate of postoperative complications in patients with OSA syndrome may be owing to a variety of reasons. Central nervous system suppression owing to anesthesia, sedation, and analgesia can foment sleepdisordered breathing. In addition, rapid eye movement (REM) sleep is diminished to absent on the first postoperative night and this typically is followed by REM rebound on subsequent nights. Due to this rebound, REM-associated hypoxemic episodes may increase 3-fold on the second and third postoperative nights, leading to an increased risk of complications." ${ }^{4}$ These questionnaires have reported high incidences of identifying those with OSA when measured by sleep studies, the gold standard for diagnosing OSA. Sleep studies can gauge mild, moderate, and severe forms of OSA., 


\begin{tabular}{|c|c|c|}
\hline $\mathrm{S}$ (snore) & Have you been told you snore? & $\mathrm{Y} / \mathrm{N}$ \\
\hline $\mathrm{T}$ (tired) & Are you often tired during the day? & $\mathrm{Y} / \mathrm{N}$ \\
\hline $\mathrm{O}$ (obstruction) & Do you know if you stop breathing while sleeping? & $\mathrm{Y} / \mathrm{N}$ \\
\hline $\mathrm{P}$ (pressure) & Do you have high blood pressure or do you take me & \\
\hline & to control high blood pressure? & $\mathrm{Y} / \mathrm{N}$ \\
\hline \multicolumn{3}{|c|}{ Answering YES to two or more STOP questions indicates you are at risk for OSA. } \\
\hline B (BMI) & Is your body mass index greater than $28 ?$ & $\mathrm{Y} / \mathrm{N}$ \\
\hline A (Age) & Are you 50 years old or older? & $\mathrm{Y} / \mathrm{N}$ \\
\hline \multirow[t]{3}{*}{$\mathrm{N}($ Neck) } & Male with neck circumference greater than 17 in & \\
\hline & or female with neck circumference greater than & \\
\hline & 16in? & $\mathrm{Y} / \mathrm{N}$ \\
\hline G (Gender) & Are you a male? & $\mathrm{Y} / \mathrm{N}$ \\
\hline \multicolumn{3}{|c|}{$\begin{array}{l}\text { The more BANG questions to which you answer YES, the greater your risk of having } \\
\text { moderate to severe OSA. }\end{array}$} \\
\hline
\end{tabular}

\section{STOP-BANG (Figure 1)}

*Page 2 of 2 of STOP-BANG research documentation.

\subsection{Sleep Architecture}

It is important to understand basic sleep architecture, or the structural organization of normal sleep, in order to manage patients with obstructive sleep apnea. These episodes disrupt the normal sleep cycle, which can cause fragmented sleep. The absence of a normal sleep cycle has been known to have serious physiological consequences.

There are two types of sleep: non-rapid eye-movement (NREM) and rapid eye-movement (REM). During the normal sleep cycle, NREM and REM alternate cyclically. According to Colten (2006), "NREM sleep is divided into stages 1, 2, 3, and 4, representing a continuum of relative depth. Each has unique characteristics including variations in brain wave patterns, eye movements, and muscle tone. Circadian rhythms, the daily rhythms in physiology and behavior, regulate the sleep-wake cycle. In addition, the sleep-wake system is thought to be regulated by the interplay of two major processes, one that promotes sleep and one that maintains wakefulness. A sleep episode begins with a short period of NREM stage 1 progressing through stage 2, followed by stages 3 and 4 and finally to REM. However, individuals do not remain in REM sleep the remainder of the night but rather cycle between stages of NREM and REM throughout the night. NREM sleep constitutes about 75 to 80 percent of total time spent in sleep, and REM sleep constitutes the remaining 20 to 25 percent. The average length of the first NREM-REM sleep cycle is 70 to 100 minutes. The second and later cycles are longer lasting, approximately 90 to 120 minutes." 8 In the normal adult, REM sleep will increase as the sleep episode increases. As the sleep episode continues, stage 2 begins to account for the majority of NREM sleep. ${ }^{8}$

The physiological changes during NREM sleep include; hypoventilation, reduced pharyngeal muscle tone, increased upper airway resistance, suppressed cough reflex, and depression of the hypoxic ventilatory response. ${ }^{8}$ Blood cortisol levels also affect REM sleep as cortisol levels are increased from the stress of surgical procedures, which decreases REM sleep time. ${ }^{5}$ The physiological changes during REM sleep include; increased heart rate, increased blood pressure, increased sympathetic nervous system activity, suppressed cough reflex, increased upper airway resistance, and increased respiration with variability and brief stoppages in ventilation. ${ }^{8}$

According to Vasu, et al (2010), "The high rate of postoperative complications in patients with OSA may be owing to a variety of reasons. Central nervous system suppression owing to anesthesia, sedation, and analgesia can foment sleep-disordered breathing and further asphyxia-related 
complications. In addition, rapid eye-movement (REM) sleep is diminished to absent on the first postoperative night, and this typically is followed by REM rebound on subsequent nights. Because of this rebound, REM-associated hypoxemic episodes may increase 3-fold on the second and third postoperative nights, leading to an increased risk of complications." ${ }^{4}$ These considerations emphasize the importance of identifying obstructive sleep apnea and the appropriate management of these patients.

\section{Materials AND Methods}

Following institutional review board (CHI Health Creighton University Medical Center in Omaha, Nebraska) approval, patients in the preoperative area were screened for study eligibility. A total of 101 subjects signed consent and HIPPA forms in order to be participants in the research study.

\subsection{Specific Aims}

To utilize an already proven screening tool (STOP-BANG) in identifying potential OSA patients. Once identified, anesthesia can evaluate if their perioperative experience can be optimized or not. All subjects were treated in the same manner as if they were not in the study; the only difference was the gathering of quantitative and qualitative data. The direction of each anesthetic was determined by the individual anesthesia provider. Intraoperatively, we assessed for difficulties with mask ventilation, direct laryngoscopy, and mechanical ventilation. Postoperatively, we assessed for difficulty extubating, marked desaturation, observed apnea, and differentiated between OSA and opioid-induced apnea. While the patient was in the recovery room, the post-anesthesia care registered nurse (PACU $\mathrm{RN}$ ) evaluated three 20-minute intervals and indicated; 1 ) if the patient desaturated by a $4 \%$ decrease for more than 10 seconds; 2) displayed periods of apnea; and 3) if the patient had pain mismatching (in which the patient indicated they were experiencing pain while demonstrating desaturation). It was also noted if positive pressure ventilation or reintubation was required and why.

\subsection{Subject Enrollment}

A study-approved anesthesia provider discussed the research study with the patient during their anesthesia preoperative evaluation. If agreed, the patient signed a consent and HIPPA authorization form. Subsequently, the STOP-BANG questionnaire was administered. Positive and negative STOPBANG questionnaires were separated and placed into positive and negative files.

\subsection{Subject Selection}

Patients had to be English speaking, at least 19 years of age, non-child bearing, capable of signing their own consent, scheduled for outpatient surgery or 24-hour observation, undergoing a general anesthetic, and agreed in writing to participate in this study. All patients were evaluated using the STOP-BANG questionnaire.

\subsection{Study Procedures}

Intraoperatively, each anesthesia provider was responsible for indicating if there was difficulty with the following: mask ventilation, direct laryngoscopy, mechanical ventilation, and/or extubation. Anesthesia providers were asked to first mask ventilate the patient; if they could not sufficiently ventilate the patient and/or required an airway to aide them, it was deemed as difficult. Any anesthesia provider who felt that a patient's laryngoscopy deviated from "easy" was deemed as difficult. The exception to difficulty with mechanically ventilating a patient is if the patient was placed in "steep" trendelenberg or high lithotomy positioning. If an anesthesia provider was unable to extubate a patient, they were instructed to indicate what they believed to be the primary issue for extended intubation. If any one of the four were experienced, for this study, it was deemed as an intraoperative complication.

Once the patient was extubated and arrived in the post anesthesia care unit (PACU), the PACU registered nurse (RN) was asked to fill out a chart, "Figure 2." The first hour of a patients' recovery was divided into three 20-minute intervals. During each 20 minutes, the RN made three assessments. First, it was noted if the patient experienced a desaturation defined by a decrease in $\mathrm{O} 2$ saturation of $4 \%$ for more than 10 seconds. Second, it was noted if the patient displayed apnea, with discretion to narcotic inducement. If the PACU RN administered opioid and believed it to be the reason for the patient's apnea, then the apnea episode was not documented. Third, it was noted if the patient was experiencing pain mismatching, which is when the patient indicated they were in pain despite 
showing signs of desaturation. In this case, the PACU RN could choose to relieve the pain with opioids but thereby risk worsening the patient's drive to adequately ventilate and potentially risk oxygen desaturation. If a patient displayed any one of the three, the nurse would place a single check in the proper box.

Having more than one check in one 20-minute interval is plausible but not significant because the patient is just emerging out of anesthesia. What is more significant is if there are checks in multiple boxes across multiple 20-minute time intervals and if a patient required a positive pressure ventilation (BiPAP/CPAP) and/or reintubation was required to necessitate recovery.

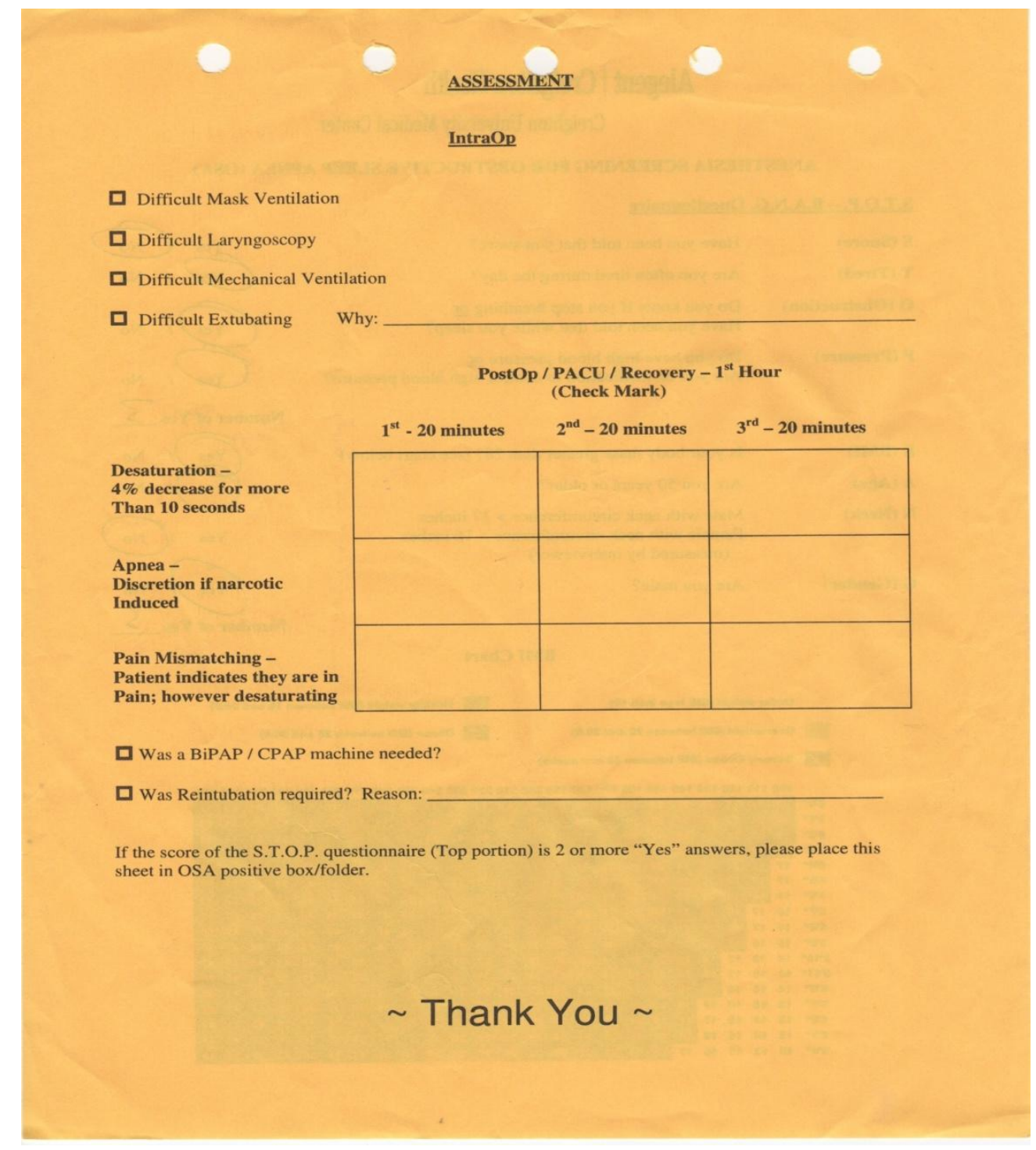

*Figure 2

*Page 2 of 2 of STOP-BANG research documentation.

\section{RESULTS}

\subsection{Statistical Analysis}

The data analysis for this paper was generated using SAS software, Version 9.4 of the SAS System for Windows. Copyright (C) 2015 SAS Institute Inc. SAS and all other SAS Institute Inc. product or service names are registered trademarks or trademarks of SAS Institute Inc., Cary, NC, USA. Variables were compared using the Chi-square test and when applicable, the Fischer exact test was used as substitute. Odds ratio and relative risks are reported with $\mathrm{p}$ values $<0.05$ considered statistically significant. The sample size for this study was determined by the number of eligible patients who provided consent. Of the 101 participants, 52 participants had positive STOP-BANG questionnaire scores and 49 participants had negative STOP-BANG questionnaire scores (See Table $1)$. 


\subsection{Intra-Operative Complications (Two or More Questions)}

Participants who answered "yes" to less than two STOP questions totaled 49 (STOP-BANG negative participants). Of the 49 participants, 47 participants did not have intra-operative complications. Only two participants who answered "yes" to less than two STOP questions experienced intra-operative complications. Participants who answered "yes" to two or more STOP questions totaled 52 (STOPBANG positive participants). Of the 52 participants, 16 participants DID have intra-operative complications ( $\mathrm{p}=0.0005) ; 36$ participants who answered "yes" to two or more STOP questions did not experience intra-operative complications. Therefore, a participant who answered "yes" to two or more STOP questions (STOP-BANG positive participants) was almost 10 times more likely to experience an intra-operative complication than a participant who answered "yes" to fewer than two STOP questions (STOP-BANG negative participants).

\subsection{PACU Complications (Two or More Questions)}

Two STOP-BANG negative participants experienced complications in the PACU whereas 47 STOPBANG negative participants experienced no complications in the PACU $(\mathrm{N}=49)$. Seven STOP-BANG positive participants experienced complications in the PACU whereas 45 STOP-BANG positive participants did not experience any complications in the PACU $(\mathrm{N}=52)$, (Two sided $\operatorname{Pr}<=0.1615)$.

\subsection{Intra-Operative Complications (Five or More Questions)}

To increase specificity, a statistical analysis was completed utilizing five "yes" STOP-BANG questions as indices for complications intra-operatively or in the PACU compared to the previous two or more/less than two participants "yes" to the STOP questions. Participants who answered "yes" to less than five STOP-BANG questions totaled 73. Of the 73 participants, seven experienced intraoperative complications compared to 66 participants that did not experience intra-operative complications. Participants who answered "yes" to five or more STOP-BANG questions totaled 28. Of the 28 participants, 11 participants experienced intra-operative complications compared to 17 participants that did not experience intra-operative complications. Therefore, a participant who answered "yes" to five or more STOP-BANG questions was six times more likely to experience an intra-operative complication than a participant who answered "yes" to less than five STOP-BANG questions (Two-sided $\operatorname{Pr}<=0.0011$ ).

\subsection{PACU Complications (Five or More Questions)}

Six participants who answered "yes" to less than five STOP-BANG questions experienced complications in PACU whereas 67 participants who answered "yes" to less than five STOP-BANG questions did not experience complications in PACU $(\mathrm{N}=73)$. Three participants who answered "yes" to five or more STOP-BANG questions experienced complications in PACU whereas 25 participants who answered "yes" to five or more STOP-BANG questions did not experience complications in PACU (N=28), (Two-sided $\operatorname{Pr}<=0.7$ ).

\subsection{BMI Analysis (Two or More Questions)}

BMI of each group -- STOP-BANG negative and STOP-BANG positive - was compared. The mean BMI for STOP-BANG negative participants was $26.3 \pm 1.6$ (95\% confidence interval). The mean BMI for STOP-BANG positive participants was $30.7 \pm 1.45$ (95\% CI). STOP-BANG positive participants had a mean BMI of $+4.4(95 \% \mathrm{CI})$ higher than STOP-BANG negative participants. This data correlates with the BMI gaps between normal-overweight and overweight-obese $(\mathrm{p}=0.0001)$.

\subsection{BMI Analysis (Five or More Questions)}

BMI was averaged between the two groups: participants who answered "yes" to less than five STOPBANG questions and participants who answered "yes" to five or more STOP-BANG questions. The mean BMI for participants who answered "yes" to less than five STOP-BANG questions was $27.0 \pm$ 1.29 (95\% confidence interval). The mean BMI for participants who answered "yes" to five or more STOP-BANG questions was $32.5 \pm 1.7(95 \% \mathrm{CI})$. The mean BMI for participants who answered "yes" to five or more STOP-BANG questions was +5.5 (95\% CI) higher than the mean BMI for participants who answered "yes" to less than five STOP-BANG questions. Again, this correlates with the BMI gaps between overweight and obese $(\mathrm{p}=0.0001)$. 


\subsection{PACU Length Of Stay}

The study also attempted to compare STOP-BANG positive and negative patients' length of stay in the PACU. PACU nurses only recorded 40 of the 104 patients' length of stay in recovery. Due to the limited documentation, this portion of the study was discarded.

Table1. Breakdown of Patients

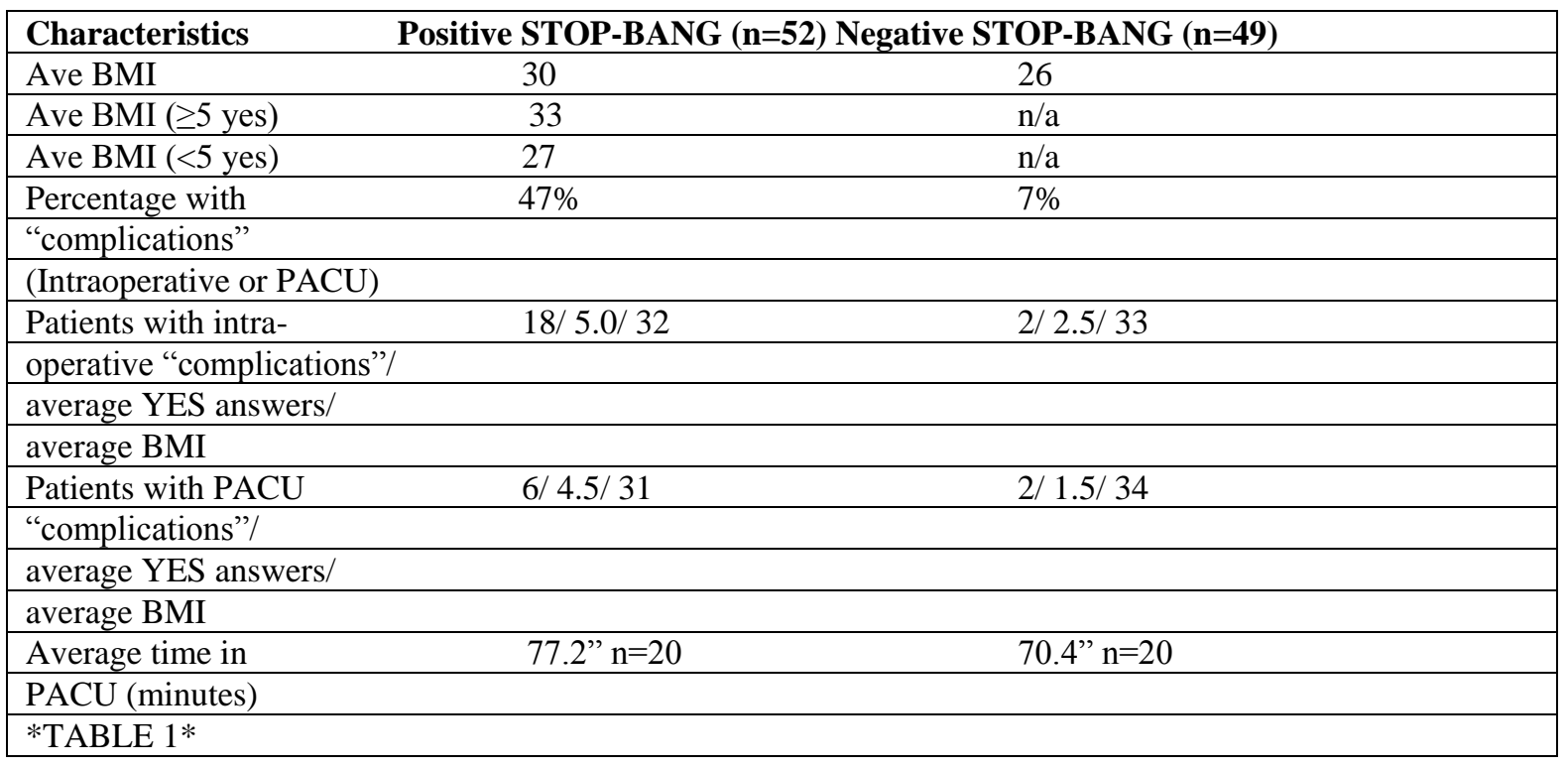

**STOP-BANG positive is designated to those who answered $\mathbf{Y E S}$ to 2 or more STOP questions.

**Intra-operative patient "complications" is defined as difficulties with mask ventilation, laryngoscopy, mechanically ventilating, and/or extubating.

**PACU patient "complications" is defined as a 4\% decrease in oxygen saturation, displaying apnea and /or desaturation, and pain mismatching; occurring in two or more 20-minute intervals.

**Limited data on length of stay in recovery room.

\subsection{Study Weaknesses}

Study weaknesses include: limited sample size, regional population, individualized general anesthetic plans and techniques, individual qualitative assessment by PACU registered nurses and limited recovery room time documentation. Further research is needed and may look at the use of regional versus general anesthetics, the use of non-respiratory depressant analgesics/anesthetics, and hemodynamic stability in patients postoperatively who are susceptible to REM sleep rebound with positive STOP-BANG questionnaires.

\section{DisCUSSION}

The purpose of this study was to demonstrate that the STOP-BANG questionnaire identifies OSA patients who are otherwise undiagnosed to prevent anesthesia-related complications intraoperatively and postoperatively. People with undiagnosed OSA undergoing anesthesia are directly and indirectly at risk for potential intraoperative and postoperative complications. People with moderate or severe OSA may unknowingly place stress on their cardio-pulmonary system, thereby depressing its function. Undiagnosed patients may potentially place themselves at higher risk for complications in the perioperative and postoperative period. Precautions may or may not have been taken because anesthesia providers did not fully assess for OSA and prepare adequately for potential airway and hemodynamic complications. Once identified, appropriate management of these patients intraoperatively and postoperatively is imperative. Regional anesthesia may be superior and associated with better outcomes compared to general anesthesia in patients with OSA as well as minimizing increased cortisol levels and its effect on sleep architecture. Another option to reduce opioids is the utilization of other pharmaceuticals, such as dexmedetomidine, ofirmev, gabapentin, ketoralac and ketalar. Anesthesia providers also should prepare for potential difficult mask ventilation and/ or difficult laryngoscopy. Postoperatively, providers could order continuous monitoring of oxygenation saturation until discharge (being aware of REM rebound on post op days 2 and 3) as well as ordering CPAP machines immediately postoperative in the PACU and for the 
duration of the patients' stay. A follow up at a sleep center for the management of sleep apnea would also be recommended at discharge. We care for many patients who fit the profile of having OSA. It is beneficial for patients to be flagged with utilization of a preoperative assessment tool such as STOPBANG. By identifying at-risk patients early, measures can be taken to increase their safety and improve their outcomes.

\section{REFERENCES}

[1] Chung F, Elsaid H. Screening for obstructive sleep apnea before surgery: why is it important. Current Opinion in Anaesthesiology 2009;(22):405-411.

[2] Gali B, Whalen FX, Scroeder DR, Gay PC, Plevak DJ. Identification of patients at risk for postoperative respiratory complications using a preoperative obstructive sleep apnea screening tool and post anesthesia care assessment. Anesthesiology. 2009;(110):869-877.

[3] Vasu T, Grewal R, and Doghramji K. Obstructive sleep apnea syndrome and perioperative complications: a systematic review of literature. Journal of Clinical Sleep Medicine JCSM 2012;8(2):199-205.

[4] Vasu T, Doghramji K, Cavallazzi R, et al. Obstructive sleep apnea syndrome and postoperative complications: clinical use of the STOP-BANG questionnaire.Arch Otolaryngol Head Neck Surg. 2010;136(10):1020-1024. doi:10.1001/archoto.2010.1020.

[5] Somers V, Dyken M, Mark A, Abboud F. Sympathetic-nerve activity during sleep in normal subjects. New England Journal of Medicine. 1993;328(5):303-307.

[6] Madsen PL, Schmidt JF, Wildschiodtz G, Friberg L, Holm S, Vorstrup S, Lassen NA. Cerebral $\mathrm{O} 2$ metabolism and cerebral blood flow in humans during deep and rapid-eye-movement sleep. Journal of Applied Physiology. 1991b;70(6):2597-2601.

[7] Henrichs B, Walsh R. Is that snoring something to worry about? Anesthetic implications for obstructive sleep apnea. American Association of Nurse Anesthetists Journal. 2012;(80):393-401.

[8] Colten, H. Sleep Physiology. In Sleep disorders and sleep deprivation an unmet public health problem. Washington, D.C.: Institute of Medicine : 2006:33-53.

[9] Carskadon M, Dement W. Normal human sleep: An overview. In: Kryger MH, Roth T, Dement WC, editors. Principles and Practice of Sleep Medicine. 4th ed. Philadelphia: Elsevier Saunders; 2005. pp. 13-23.

[10] NHLBI (National Heart, Lung, and Blood Institute) Sleep, Sleep Disorders, and Biological Rhythms: NIH Curriculum Supplement Series, Grades 9-12. Colorado Springs, CO: Biological Sciences Curriculum Study; 2003. 